\title{
Knowledge Mapping of Researches on China's Aphids Parasitoids Based on Metrology Analysis with CiteSpace V
}

\author{
Chao Zhang ${ }^{1, \mathrm{a}}$, Changzhong Liu ${ }^{2, \mathrm{~b}, *}$ \\ ${ }^{1}$ College of Pratacultural Science, Gansu Agricultural University, Lanzhou, Gansu Province, China \\ ${ }^{2}$ College of Plant Protection, Gansu Agricultural University, Lanzhou, Gansu Province, China
}

\begin{abstract}
China's aphids parasitoids from 1979 to 2018 years were taken from the China National Knowledge Infrastructure as the research samples, which were analyzed using CiteSpace V as an analysis tool, to create a knowledge map on the research fields of China's aphids parasitoids and comprehensively analyze the number of publications, core authors and research institutions, research hot topics and research trends, so as to present the current research status of China's aphids parasitoids and provide a reference for the future researches.
\end{abstract}

\section{Introduction}

Aphids mainly live in temperate regions. There are more than 4,700 species known in the world, over 1,000 species of which are living in China ${ }^{[1-2]}$. Aphids have strong adaptability to environmental conditions. At the same time they not only harm the economic plants, but also spread plant viruses, so how to effectively control them has become a major issue that many researchers have been working on ${ }^{[1]}$. In recent years, the research on the control technology of aphids has entered a new age, and the biological control of natural enemy is the development trend. The main parasitic natural enemy of the aphids is aphidius belonging to Hymenoptera Ichneumonoidea. These species of parasitic wasps can significantly control the number of aphids ${ }^{[3]}$.A large number of researches on the biological characteristics, parasitic environmental conditions, parasitic selection behaviors and co-evolution with host of aphidius have been conducted in foreign countries ${ }^{[4-7]}$. Although China has abundant resources of aphidius, the researches on aphidius are few. The research on China's aphidius and its application have not only become a hot spot in pest biological control and plant protection research, but also a key and difficult point to be solved urgently in the practice of agricultural production ${ }^{[8]}$.

Based on the literature of the CNKI journal database, this paper uses the information visualization software CiteSpace V to draw knowledge map, conducts quantitative analysis on the research hot spots, research frontiers and research trend of Chinese aphidius, and clarifies the research status, development context and future trend of China's aphidius, to summarize the research dynamics of this topic, and provide a new idea for the exploration of the research frontiers and hotspots of aphidius and a reference for researchers to further

\footnotetext{
a Corresponding author: liuchzh@gsau.edu.cn
}

study of aphidius.

\section{Data source and research methods}

\subsection{Data source}

The data are from CNKI journal database, the search strategy is "(topic = aphidius) (accurate matching)", the time period is from January 1, 1979 to June 20, 2018, a total of 722 papers are retrieved, and 554 valid documents are obtained by removing the literature that cannot meet the requirements.

\subsection{Research methods}

Knowledge map is a method to visually display the knowledge context of a subject by using data information analysis, quantization and graph drawing ${ }^{[9]}$. This paper applies CiteSpace V, an information visualization software developed by Professor Chen Chaomei, as an analysis tool, uses CiteSpace V to draw the keyword map and the Timeline view ${ }^{[10]}$. This paper analyzes the number of published papers, core authors and major research institutions by using bibliometric analysis method and co-word analysis method, describes the research hotspots, research frontiers and research trend of Chinese aphidius based on the mapped knowledge map and analyzes the research status of China's aphidius and its development context.

\section{Overview of research on Aphidius}

\subsection{Number of published papers}

It can be seen from Figure 1 that the number of published 
papers on China's aphidius rised generally. The study began in 1979, the number of publications increased rapidly from 1980 to 1989 , with a maximum of 12 papers, related studies from 1990 to 1995 were fewer, the attention on the research on aphidius from 1999 to 2007 increased, and there had been an increase in research on the control of wheat aphid by aphidius. The research on aphidius has entered a new era since 2009, especially under the guidance of the national policy of reducing the use of chemical pesticide and the impact of market environment strongly demanding green products, the focus on the research on aphidius increased, and the number of papers on using aphidius to control Myzus persicae swiftly increased. By 2017, the maximum number of annual publications reached 47 , about 4 times of that in 2009. Since the statistical time ends on June 20, 2018 , the number of documents issued in 2018 decreased.

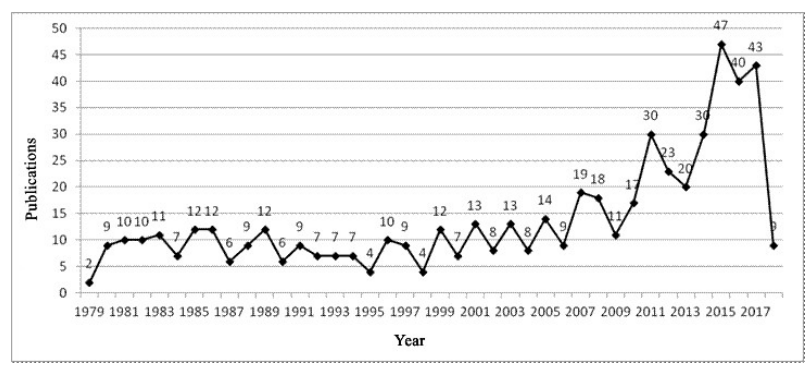

Figure 1. The number of published papers on domestic aphids parasitoids research in 1979-2018

\subsection{Core authors}

The maximum number of papers published on China's aphidius by one author is up to 14 pieces. According to the calculation of Price's law ${ }^{[11]}$, the authors publishing more than 3 papers are core authors. Combined with the results of quantitative analysis, there are 151 core authors. It can be seen from Table 1 that there are 6 authors who published more than 11 papers on China's aphidius, including Jiahua Chen, Wenlong Chen, Shusheng Liu, Xinghui Gu, Shuoyuan Yang and Zhengduan Ji.

Table 1 prolific authors of aphids parasitoids research in 1979-2018 (6 or above)

\begin{tabular}{lc|lc}
\hline Authors & Publications & Authors & Publications \\
\hline Jiahua Chen & 14 & Hongrui Zhang & 7 \\
Wenlong Chen & 14 & Xinming Yin & 7 \\
Shusheng Liu & 12 & Qinlei Meng & 7 \\
Huixing Gu & 11 & Chunming Liu & 7 \\
Shuoyuan Yang & 11 & Chao Wang & 7 \\
Zhengduan Ji & 11 & Yufang Yan & 7 \\
Limeng Zhang & 10 & Aiping Liu & 7 \\
Yong Zhang & 10 & Kun Huang & 6 \\
Wei Wu & 9 & Xingfu Wu & 6 \\
Jianhua Deng & 8 & Jian Zhu & 6 \\
\hline
\end{tabular}

\subsection{Main research institutions}

As can be seen from Table 2, Institute of Entomology in Guizhou University published 12 papers, which is the largest number. Institute of Zoology in Chinese Academy of Sciences, Yunnan Province Yuxi Tobacco Company and Hebei Agricultural University are also the main forces of the study on aphidius, for they published 11 papers respectively. College of Agriculture and Biotechnology in Zhejiang University, Kunming Institute of Zoology in Chinese Academy of Sciences and College of Plant Protection in Yunnan Agricultural University respectively published 10 papers. College of Plant Protection in Shangdong Agricultural University published 9 papers. Chinese Academy of Sciences, tobacco companies and agricultural universities are at the forefront of research in this field. In addition, agricultural technology companies and forestry colleges have also achieved some research results.

Table 2 Top 18 institutions of aphids parasitoids research in 1979-2018

\begin{tabular}{|c|c|c|c|}
\hline Institutions & Publications & Institutions & Publications \\
\hline $\begin{array}{l}\text { Institute of Entomology, } \\
\text { Guizhou University }\end{array}$ & 12 & $\begin{array}{l}\text { Yunnan province } \\
\text { Tobacco Company }\end{array}$ & 8 \\
\hline $\begin{array}{l}\text { Institute of Zoology, } \\
\text { Chinese Academy of } \\
\text { Sciences }\end{array}$ & 11 & $\begin{array}{l}\text { Anhui } \\
\text { Agricultural } \\
\text { University }\end{array}$ & 7 \\
\hline $\begin{array}{l}\text { Yunnan province Yuxi } \\
\text { Tobacco Company }\end{array}$ & 11 & $\begin{array}{l}\text { Yunnan province } \\
\text { Honghe Tobacco } \\
\text { Company }\end{array}$ & 7 \\
\hline $\begin{array}{l}\text { Hebei Agricultural } \\
\text { University }\end{array}$ & 11 & $\begin{array}{l}\text { College of Plant } \\
\text { Protection, Fujian } \\
\text { Agriculture and } \\
\text { Forestry } \\
\text { University }\end{array}$ & 6 \\
\hline $\begin{array}{lr}\text { College } & \text { of } \\
\text { and } & \text { Biotechnolture } \\
\text { Zhejiang } & \text { University } \\
\text { Collet } & \end{array}$ & 10 & $\begin{array}{l}\text { College of Plant } \\
\text { Protection, Henan } \\
\text { Agricultural } \\
\text { University }\end{array}$ & 6 \\
\hline $\begin{array}{l}\text { Kunming Institute of } \\
\text { Zoology, Chinese } \\
\text { Academy of Sciences }\end{array}$ & 10 & $\begin{array}{l}\text { Institute of } \\
\text { Grassland } \\
\text { Research of } \\
\text { Chinese Academy } \\
\text { of Agricultural } \\
\text { Sciences }\end{array}$ & 5 \\
\hline $\begin{array}{l}\text { College of Plant } \\
\text { Protection,Yunnan } \\
\text { Agricultural University }\end{array}$ & 10 & $\begin{array}{l}\text { Kunming Runcai } \\
\text { Agricultural } \\
\text { Technology } \\
\text { Development Co., } \\
\text { Ltd. }\end{array}$ & 5 \\
\hline $\begin{array}{l}\text { College of Plant } \\
\text { Protection, Shandong } \\
\text { Agricultural }\end{array}$ & 9 & $\begin{array}{l}\text { Southwest } \\
\text { Forestry } \\
\text { University }\end{array}$ & 5 \\
\hline $\begin{array}{l}\text { College of plant } \\
\text { protection, Northwest } \\
\text { Agriculture and Forestry } \\
\text { University }\end{array}$ & 8 & $\begin{array}{l}\text { Fujian } \\
\text { Agricultural } \\
\text { College }\end{array}$ & 5 \\
\hline
\end{tabular}

\section{Research hotspots and research trend} of Aphidius

\subsection{Analysis on the research hotspots of Aphidius}

Through operating CiteSpace V, keyword map of the 
aphidius research field is obtained, including 741 nodes and 971 connections. As is shown in Figure 2, the intermediary centrality reflects the relationship between a node and other nodes. The node with high intermediary centrality is usually located between two different clusters, that is, it can reflect the research hotspots and frontiers in this research field ${ }^{[10]}$. In Figure 2, the nodes with purple outer ring have higher intermediary centrality. The larger the node, the higher the frequency at which the key word appears. The clustering nodes in the figure form into a obvious circle, and the biological control applying aphidius has become a research hotspot. The nodes of the top 7 intermediary centrality (intermediary centrality $\geq 0.10$ ) in the map are: Aphidius gifuensis $(0.78,204)$ ), aphidius $(0.32,106)$, biological control $(0.12,37)$, parasitic rate $(0.2,32)$, Ephedrus plagiator $(0.12,26)$, Sitobion avenae $(0.13,24)$ and aphidiidae $(0.16,18)$. The above key nodes are the research hotspots of China's aphidius (numbers in the brackets are the intermediate centrality and the frequency of occurrence of the key words).

A. gifuensis is a hot spot of the studies on China's aphidius, and parasitic rate are the focus of applying aphidius to conduct biological control. Aphidiidae, Diaeretiella rapae and $S$. avenae, these three key words with high intermediary centrality, are within the research area of biological control. China's focus on promoting the ecological development of green agriculture and other series of action plans further promote the development of biological control, and biological control research on applying parasitic pests of aphidius has become one of the research hotspots. From the results of key word measurement in the mapping process, it can be found that key words related to research on aphidius such as $A$. avenae $(17,0.08)$, host plant $(10,0.08)$, biology $(10,0.07)$, propodeum $(18,0.05)$. control effects $(10,0.07)$, eclosion rate $(17,0.08)$, Schizaphis graminum $(12,0.04)$ and population dynamic $(17,0.08)$ are all high-frequency words.

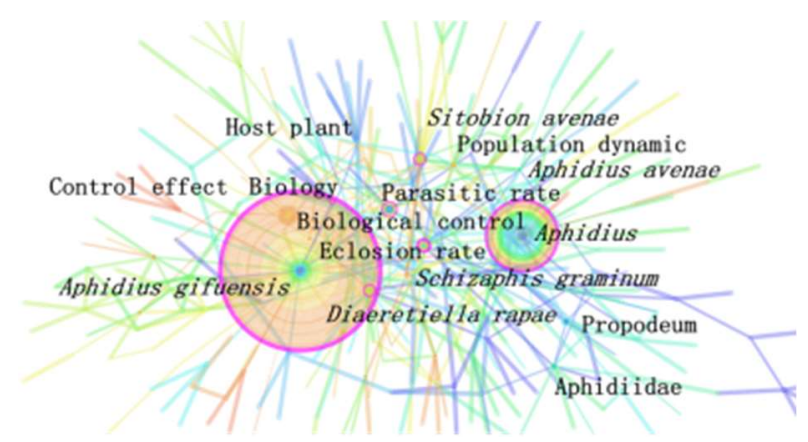

Figure 2. The keywords of aphids parasitoids research in 1979-2018

\subsection{Research trend of Aphidius}

The time zone map of the development trend of China's aphidius is obtained by operating CiteSpace V. Figure 3 shows the ideological state of the research field of China's aphidius, through which the development trend of aphidius can be observed.
The research on aphidius entered the early stage of development in 1979. By the end of 1987, scholars mainly studied various species of common aphidiuses in China, including parasitic wasps like E. plagiator, D. rapae, $A$. gifuensis and $A$. avenae and natural enemy parasitic wasps of crop pest as $S$. graminum, and explored their biological characteristics such as spawning organs, occurrence regularity, developmental duration, developmental rate, host age and parasitism rate.

With the deepening of basic research, scholars have explored the feasibility of pest control in 1988. The main concern was the natural enemies of aphid such as the Lysiphlebus japonicus, study the influence of environmental factors like photoperiod and application of imidacloprid on the community structure and population dynamics of the aphidius. With the rising application of greenhouse cultivation, the Trialeurodes vaporariorum sucks the juice of greenhouse products including vegetables, flowers and herbs, making the leaves fade, wilted or even dead, and causing the harm of sooty mould and spreading viral diseases to become more serious. In 2000 , the studies of the aphidius mainly focused on applying insecticide spray and Encarsia formosa to control the $T$. vaporariorum, and explored its prevention and treatment effects. Studies have shown that $A$. gifuensis is an important parasitic natural enemy of tobacco aphid. however, the manipulative affect of $A$. gifuensis to aphid is greatly influenced by the hyperparasitioid. In 2003, scholars studied the biological characteristics and behavioral characteristics of Pachyneuron aphidis, which had good practical value for improving the reproductive utilization rate of $A$. gifuensis. In the same year, there are also related reports on the influence of pesticide application on the detoxification enzymes of aphidius. In 2003-2009, a series of studies by aphidius on Aphis craccivora by scholars from the Chinese Academy of Sciences and Nanjing Agricultural University made it one of the research hotspots at that time. With the development of animal husbandry in China, the pest control of alfalfa and other pastures has gradually become a research hotspot. A. craccivora is a key pest of the pasture industry in China, which brings great economic losses to the animal husbandry. Since 2012, some scholars have carried out research on the influence of basic biology and parasitic factors on L. testaceipes, an important kind of parasitic wasp of $A$. craccivora that is introduced into China from Oklahoma, USA, and also explored indoor breeding technology, which provide a scientific basis for the biological control of $A$. craccivora. In 2015, domestic scholars studied the application of $A$. ervi in controlling Acyrthosiphon pisum. Subsequently, scholars have made some progress by combining pest control with plant-derived pesticides and ladybirds. $A$. gifuensis is the dominant parasitic natural enemy of $M$. persicae, and have a good control effect. In 2018, scholars analyze and discuss the factors affecting the various aspects of the technology and propose measures to optimize the effect of applying floating bed seeding production to breed $A$. gifuensis, which provide the basis and technical support for the wider application of it. 


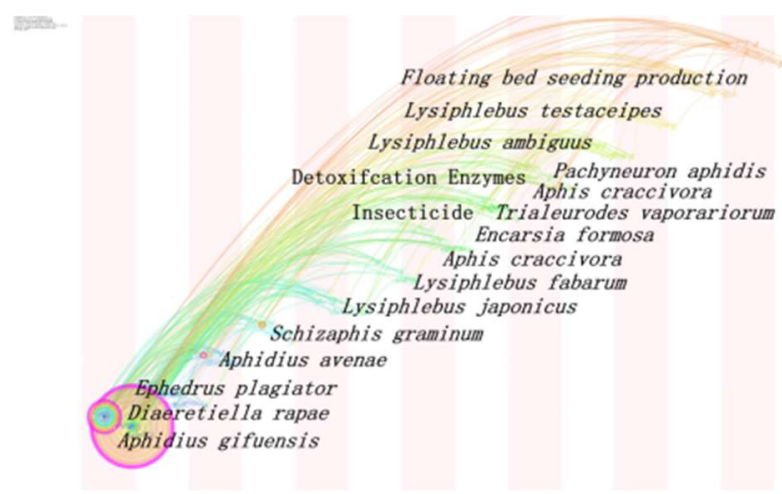

Figure 3. The timeline of aphids parasitoids research in $1979-2018$

\section{Conclusion}

This paper uses the information visualization software CiteSpace V as an analysis tool, analyzes 554 effective scientific and technical documents collected by CNKI from 1979 to 2018, and obtains the following conclusions.

The number of published papers on aphidius in China is generally on the rise. There are many core authors in the study, but the amount of papers is not large. Chinese Academy of Sciences, tobacco companies and agricultural universities are at the forefront of research in this field. The research hotspots of China's aphidius mainly include A. gifuensis, aphidius, biological control, parasitic rate, $D$. rapae, $S$. avenae and aphidiidae. The study of the aphidius has a long duration and also has continuity, for it has been extended from basic biological research and ecological research to the application of biological control in tobacco and other industries. China's scholars explored the feasibility of using aphidius to control pests, and studied the effect of environmental factors on the community structure and population dynamics of aphidius. It can be seen that the integrated control such as combining aphidius with non-chemical pesticides is the future research trend of aphidius.

This paper uses the CiteSpace $\mathrm{V}$ information visualization software to draw knowledge map for the research status, progress and trend of China's aphidius field for the first time, and then conducts quantitative analysis on it. As the CiteSpace V software cannot process documents from multiple sources at the same time, the data source only uses the CNKI library. Moreover, CNKI has no relevant data on the citations of the paper for the time being, so this paper does not conduct citation analysis on the research of aphidius. In the future research work, the author will try to analyze other libraries, introduce citation analysis, and compare the research situation of the aphidius at home and abroad, in order to more comprehensively propose the strategies for developing the research on China's aphidius.

\section{References}

1. Y.L. Ma, and C.Z. Liu. Review on ecological characteristics and control of aphids. Pratacultural Science, vol.31, pp.519-525, 2014.

2. P.H. Tang, G.P. Chen, M.K. Zhu, L.J. Ren and Z.L. Hu. Advances in aphid control technology. Plant Protection, vol.39, pp.5-12+19, 2013.

3. J.H. Chen and Q.X. Shi. Sestematic studies on aphidiidae of China ( Hymenoptera:Aphidiidae). Fujian Science and Technology Publishing house, pp.1-31, 2001.

4. X.Z. He, Q. Wang and D.A.J. Teulon. Host age preference behavior in Aphidius ervi Haliday (Hymenoptera: Aphidiidae). Journal of Insect Behavior, vol.24, pp.447-455, 2011.

5. J.L. Kugler and R.H. Ratcliffe. Resistance in alfalfa to the red form of the pea aphid (Homoptera: Aphididae). Journal of Economic Entomology, vol.76, pp.74-76, 1983.

6. S.A. Langley, K.J. Tilmon, B.J. Cardinale and R.I. Anthony. Learning by the parasitoid wasp, Aphidius ervi (Hymenoptera: Braconidae), alters individual fixed preferences for pea aphid color morphs. Oecologia, vol.150, pp.172, 2006.

7. F. Pennacchio, M.C. Digilio and E. Tremblay. Biochemical and metabolic alterations in Acyrthosiphon pisum parasitized by Aphidius ervi. Archives of Insect Biochemistry \& Physiology, vol.30, pp.351-367, 2010.

8. X.Y. Ji. Impact factors for the parasitization of Microplitis and the influence of parasitzation on host biochemical metabolism. Hunan Agricultural University, pp.1-14, 2012.

9. R.M. Shiffrin and K. Börner. Mapping knowledge domains. Proceedings of the National Academy of the USA, vol.101, pp.5183-5185, 2004.

10. Y. Chen, C.M. Chen, Z.G. Hu and X.W. Wang. Principles and Applications of Analyzing a Citation Space. Science Press, pp.1-163, 2014.

11. W. Zhong, Evaluation about the Core Authors Based on Price Law and Comprehensive Index Method --Take Journal of Library Development as an Example, Science \& Technology Management Research, vol.32, pp.57-60, 2012. 